IBIMA Publishing

International Journal of Case Reports in Medicine

http://www.ibimapublishing.com/journals/IJCRM/ijcrm.html

Vol. 2015 (2015), Article ID 620549, 4 pages

DOI: $10.5171 / 2015.620549$

Case Report

\title{
Carotid Sinus Syndrome In A Patient With Hodgkin's Lymphoma
}

\section{Ricardo Humberto de Miranda Félix ${ }^{1}$, Ana Rita Brito Medeiros da Fonseca ${ }^{2}$ Beatriz H C S Silva ${ }^{3}$ and Aécio Flávio Teixeira Góis ${ }^{4}$}

\author{
Department of Internal Medicine, Federal University of São Paulo, R. Napoleão de Barros, 715, São \\ Paulo, Brazil
}

Correspondence should be addressed to: Ricardo Humberto de Miranda Félix; ricardoh_medicina@yahoo.com.br

Received date: 18 April 2014; Accepted date: 3 September 2014; Published date: 16 October 2015

Academic Editor: Božo Krušlin

Copyright (C) 2015. Ricardo Humberto de Miranda Félix, Ana Rita Brito Medeiros da Fonseca Beatriz H C S Silva and Aécio Flávio Teixeira Góis. Distributed under Creative Commons CC-BY 4.0

\begin{abstract}
Carotid sinus syndrome is an exaggerated response to carotid sinus baroreceptor stimulation and has been reported in patients with cancer. Here we present a 22-year-old man diagnosed with Hodgkin's lymphoma and admitted with pulmonary sepsis. He evolved with hypotension, bradycardia and syncope after infection control. A CT scan of neck revealed nodal compression of the right common carotid artery. The patient improves after transvenous cardiac pacing and chemotherapy.
\end{abstract}

Keywords: Carotid sinus syndrome, Hodgkin's lymphoma, chemotherapy.

\section{Introduction}

Carotid sinus syndrome (CSS) is an exaggerated response to carotid sinus baroreceptor stimulation. It tends to occur in older patients and is usually responsible for recurrent episodes of syncope, bradycardia and asystole (Moya et al (2009); Humm et al (2006)). This condition is life threatening unless promptly recognized and treated. We report a case of Hodgkin's lymphoma leading to CSS.

\section{Case Report}

A 22- year-old man presented with a 12month history of weight loss, itching, heavily night sweating and cervical lymph node enlargement. Excisional lymph node biopsy from the neck revealed nodular sclerosing Hodgkin's Lymphoma. Immunohistochemical staining turned out to be positive for CD20, CD30, BCL6, PAX5 and negative for CD3, CD15, EMA, EBV, LCA, ALK. After one month he was admitted due to respiratory failure and sepsis. Soon after admission the patient was intubated for mechanical ventilatory support. Broadspectrum antibiotics and vasoactive drugs were started. The patient did well and was extubated a week later. However, he evolved with increasing neck enlargement, bradycardia, transient hypotension and syncope with no neurological deficit. Echocardiogram was normal and electrocardiogram revealed sinusal bradycardia. Axial CT image obtained proximal to the bifurcation of the right common carotid artery shows obliteration of the perivascular fat planes with

Cite this Article as: Ricardo Humberto de Miranda Félix, Ana Rita Brito Medeiros da Fonseca Beatriz H C S Silva and Aécio Flávio Teixeira Góis (2015), "Carotid Sinus Syndrome In A Patient With Hodgkin's Lymphoma ", International Journal of Case Reports in Medicine, Vol. 2015 (2015), Article ID 620549, 
displacement and narrowing of the adjacent right jugular vein due to nodal compression (Figure 1). CSS was considered and atropine was administered without response. Transvenous cardiac pacing was inserted and dexamethasone pulse therapy $(40 \mathrm{mg} /$ day for consecutive
4 days) was started along with chemotherapy (doxorubicin, bleomycin, vinblastine, and dacarbazine). After 4 days the chemotherapy reduced the tumor mass and the patient was extubated. Pacemaker was removed soon after that and the patient was discharged.

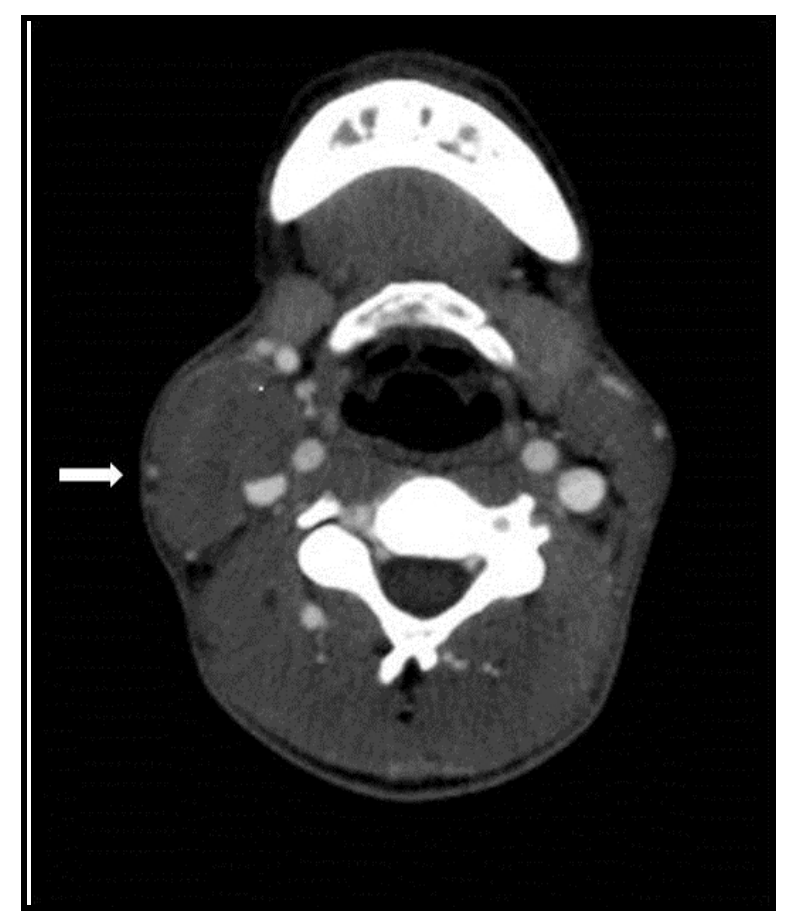

Figure 1: A contrast CT scan at the level of the bifurcation of the right common carotid artery shows large nodal mass extending into the carotid space (arrow)

\section{Discussion}

The CSS consists of a cardiovascular symptom complex resulting from excitation of a hyperactive carotid sinus reflex (Hong et al (2000)). It has been reported in patients with head and neck malignancies, paraganglioma and peripheral nerve tumor (Arapakis et al (2004)). To our knowledge, this is the first report of CSS caused by Hodgkin's lymphoma (Arapakis et al (2004); Ballantyne et al (1975); Rusconi et al (1996); Lopez et al (2000); Venkatraman et al (2005); Takahashi et al (2006)).

The carotid sinus is a dilated segment of the internal carotid arteries, located just superior to the bifurcation of the common carotid artery. Within it arterial adventitia, there are high pressure baroreceptors responsible to regulate blood pressure and heart rate. These receptors are sensitive to stretching of the arterial wall and gives rise to sensory impulses carried via the Sinus Nerve of Hering (which is a branch of the glossopharyngeal nerve) to the nucleus tractus solitarii in the medulla oblongata. The efferent limb of the reflex is carried via vagus and cervical sympathetic nerves to the heart and vessels, controlling heart rate and vasomotor tone (Hong et al (2000); Seifer (2013); Sharma et al (2011)).

When the mean arterial pressure increases, the walls of these vessels passively expand, stimulating firing of these receptors. Stimulation of baroreceptors increases vagal activity and inhibits sympathetic activity. If arterial blood pressure suddenly falls, decreased stretch of the arterial walls leads to a decrease in receptor firing (Hong et al (2000)). 
Injury of this sensitive autonomic system is the pathophysiological basis of CSS in our case and other head and neck malignancies. Its etiology is multifactorial, including a mechanical compression of the carotid sinus by tumor mass, impairing blood flow and causing reflex hypersensitization; in addition to compression of glossopharyngeal nerve, which passes between the internal jugular vein and internal carotid artery, creating a triggering focus that renders the entire arc hypersensitive. Other mechanism proposed is the infiltration of the carotid sinus resulting in increased activity of the Hering's nerve and in the parasympathetic arm of the reflex arc (Hong et al (2000); Lopez et al (2000); Takahashi et al (2006)). It cannot be proved in our case because a histopathological study of the vessel was not performed.

It is also described that depolarization of axons caused by tumor mass neighboring the carotid sinus, with a tendency to stimulate adjacent uninjured axons, can cause CCS (Sobol et al (1982); Hong et al (2000)).. The tumor may cause either a spontaneous abnormal afferent discharge in the damaged nerve itself or may lead to ephaptic conduction, either between glossopharyngeal efferent motor fibers and afferent sinus sensory fibers, or between the glossopharyngeal nerve and other nearby nerves, inducing an abnormally strong carotid sinus reflex (Noroozi et al (2012)).

CSS can be classified according to three types of response to carotid sinus massage: cardioinhibitory carotid sinus hypersensitivity, diagnosed by a greater than or equal to 3-second pause; a vasodepressor response, with the reduction in blood pressure of at least 50 $\mathrm{mm} \mathrm{Hg}$ in the absence of significant bradycardia; and a third subtype, secondary to the combination of these two mechanisms (Seifer (2013)). There is a transient reduction in cerebral perfusion triggering dizziness and syncope (Seifer (2013); Lopez et al (2000)). In our case there is a mixed response of cardioinhibitory and vasodepressor mechanisms resulting in the combination of bradycardia and peripheral vasculature vasodilatation with hypotension. Syncope was a mild symptom probably because the patient was bedridden while admitted.

The initial treatment includes anticholinergic medications, such atropine, and temporary cardiac pacing, but the definite therapy should always address the underlying cause of CSS (Arapakis et al (2004); Hong et al (2000); Lopez et al (2000); Venkatraman et al (2005); Takahashi et al (2006); Tulchinsky et al (1988)).

\section{References}

1. Arapakis, I, Fradis, M, Ridder, G. J., Schipper, J. and Maier, W. (2004) "Recurrent syncope as presenting symptom of Burkitt's lymphoma at the carotid bifurcation," Annals of Otology, Rhinology \& Laryngology, 113 373-377.

2. Ballantyne, F. III.,VanderArk, C. R. and Hilick, M. (1975) "Carotid sinus syncope and cervical lymphoma," Wisconsin Medical Journal, 74 91-92.

3. Hong, A. M., Pressley, L. and Stevens, G. N. (2000) "Carotid sinus syndrome secondary to head and neck malignancy: case report and literature review," Clinical Oncology, 12 (6) 409-412.

4. Humm, A. M. and Mathias, C. J. (2006) "Unexplained syncope--is screening for carotid sinus hypersensitivity indicated in all patients aged $>40$ years?," Journal of Neurology, Neurosurgery \& Psychiatry, 77 1267-1270.

5. Lopez, F. F., Mangi, A. A., Strenger, R, Schiffman, F. J. and Meringolo, R. D. (2000) "B-cell bradycardia: carotid sinus massage by a high-grade lymphoma," American Journal of Hematology, 64 (3) 232.

6. Moya, A, Sutton, R, Ammirati, F, Blanc, J, Brignole, M, Dahm, J. B. et al. (2009) "Guidelines for the diagnosis and management of syncope (version 2009): The Task Force for the Diagnosis and Management of syncope of the European Society of Cardiology (ESC)," European Heart Journal, 30 2631-2671.

7. Noroozi, N, Modabber, A, Hölzle, F, Braunschweig, T, Riediger, D, Gerressen M. et al. (2012) "Carotid sinus syndrome as

Ricardo Humberto de Miranda Félix, Ana Rita Brito Medeiros da Fonseca Beatriz H C S Silva and Aécio Flávio Teixeira Góis (2015), International Journal of Case Reports in Medicine, DOI: 10.5171/2015. 620549 
the presenting symptom of cystadenolymphoma," Head \& Face Medicine, 831.

8. Rusconi, L. and Zenchi, G. P. (1996) "Carotid sinus syndrome associated with a left parapharyngeal non-Hodgkin's lymphoma," Giornale Italiano di Cardiologia, 26 681-687.

9. Sharma, J. and Dougherty, A. H. (2011) "Recurrent Syncope in a Cancer Patient: A Case Report and Review of the Literature," Cardiology Research and Practice, 2011 (4) 1-5.

10.Seifer, C. (2013) "Carotid sinus syndrome," Cardiology Clinics, 31 (1) 111121.

11.Takahashi, T, Obata, N, Sugawara, N, Fujita, K, Oikawa, K, Yoshimoto, M, et al.
(2006) "Carotid sinus syncope in a patient with relapsed cervical lymphoma," International Journal of Hematology, 84 (1) 92-93.

12.Sobol, S.M., Wood B.G., Conoyer, J.M. (1982) "Glossopharyngeal neuralgiaasystole syndrome secondary to parapharyngeal space lesions," Otolaryngology - Head and Neck Surgery," 90 16-19.

13.Tulchinsky, M. and Krasnow, S. H. (1988) "Carotid sinus syndrome associated with an occult primary nasopharyngeal carcinoma," Archives of Internal Medicine, 148 (5) 1217-1279.

14. Venkatraman, V, Lee, L. and Nagarajan, D. V. (2005) "Lymphoma and malignant vasovagal syndrome," British Journal of Haematology, 130323. 